\title{
Neural interfaces based on flexible graphene transistors: A new tool for electrophysiology
}

\author{
A. Guimerà-Brunet ${ }^{1,2}$, E. Masvidal-Codina ${ }^{1}$, X. Illa $^{2,1}$, M. Dasilva ${ }^{3}$, A. Bonaccini-Calia ${ }^{4}$, E. Prats-Alfonso ${ }^{2,1}$, J. Martínez- \\ Aguilar ${ }^{1,2}$, J.M. De la Cruz ${ }^{4}$, R. Garcia-Cortadella ${ }^{4}$, N. Schaefer ${ }^{4}$, A. Barbero ${ }^{3}$, P. Godignon ${ }^{1}$, G. Rius ${ }^{1}$, E. Del Corro ${ }^{4}$, J. \\ Bousquet $^{4}$, C. Hébert ${ }^{4}$, R. Wykes ${ }^{5}$, M.V. Sanchez-Vives ${ }^{3,6}$, R. Villa ${ }^{1,2}$ and J.A. Garrido ${ }^{4,6}$ \\ ${ }^{1}$ Institut de Microelectrònica de Barcelona, IMB-CNM (CSIC), Esfera UAB, Bellaterra, Spain. email: anton.guimera@csic.es \\ ${ }^{2}$ Centro de Investigación Biomédica en Red en Bioingeniería,Biomateriales y Nanomedicina (CIBER-BBN), Madrid, Spain. \\ ${ }^{3}$ Institut d'Investigacions Biomèdiques August Pi i Sunyer (IDIBAPS), Barcelona, Spain. \\ ${ }^{4}$ Catalan Institute of Nanoscience and Nanotechnology (ICN2), CSIC and The Barcelona Institute of Science and Technology (BIST), \\ Campus UAB, Bellaterra, Barcelona, Spain. email: joseantonio.garrido@icn2.cat \\ ${ }^{5}$ UCL Institute of Neurology, University College London, Queen Square, London \\ ${ }^{6}$ Institució Catalana de Recerca i Estudis Avançats (ICREA), Barcelona, Spain.
}

\begin{abstract}
The use of graphene transistors for transducing neural activity has demonstrated the potential to extend the spatiotemporal resolution of electrophysiological methods to lower frequencies, providing a new tool to understand the role of the infra-slow activity.
\end{abstract}

\section{INTRODUCTION}

The brain is one of the most elaborate structures in nature, its activity spans over multiple temporal and spatial scales that requires a set of technologies to advance in its understanding. Through the years, the advance in neuroscience has benefited from coordinated and interdisciplinary efforts from different fields such us genetics, immunostaining, electrophysiology, optical or computational tools, which has made possible to identify neuronal types, the principles of neural coding, or the role of specific regions of the brain [1].

Electrophysiology is consistently used to measure the brain activity due to the electrical nature of neuronal activity. Typically, electrophysiological methods can be used to measure the activity at different spatial resolutions. At the microscale, patch-clamp techniques are used to measure from currents of single ion channels to single neuron activity. At the macroscale, electroencephalography (EEG) detects spontaneous or evoked electrical activity from the scalp with low spatial resolution (cm range) due to the skull's filtering [2], [3]. Large areas of the brain can also be observed with indirect measurements of the brain's activity via functional magnetic resonance imaging (fMRI), positron emission tomography (PET), or magnetoencephalography (MEG), resolving functional connectivity among brain regions. At the mesoscale, extracellular recordings enable simultaneous measuring of local field potentials (LFPs) from a population of neurons and extracellular action potentials (EAPs) from single cells. Electrocorticography $(\mathrm{ECoG})$ is the current solution to obtaining large area brain recordings with high temporal resolution. $\mu \mathrm{ECoG}$ consists in placing microelectrode arrays (MEAs) directly on the cortex surface and records signals from a large number of cortical columns [4]. In addition, the deeper activity along the cortical columns are commonly recorded via implants inserted in the cortex or deeper structures [5].
To implement any electrophysiology method an efficient transducer is required. The voltage drop caused by ionic transmembrane currents in the surrounding extracellular medium must be converted into an electrical signal to be recorded by the electronic instrumentation. Passive metallic electrodes are the most commonly used transducers [6]. The advance of the microfabrication techniques together with the investigation in novel materials (porous metals, oxides, or conductive polymers) lead to the integration of large number of recording sites in the neural interfaces, improving the spatial resolution while preserving the signal-to-noise ratio [4], [5], [7].

Thanks to their unique set of properties, graphene is considered as a transducer with very high potential for next generations of neural interfaces. Graphene exhibits high carrier mobility and field effect properties [8], enabling the implementation of the so-called graphene based solution-gated field-effect transistor (gSGFET) as a transducer for neural signals. The intrinsic local amplification of this configuration reduces the sensitivity to external noise sources. The high chemical stability [9] allows the operation without a gate dielectric layer, increasing the gate capacitance and therefore the transistor transconductance [10]. The use of a transistor as a transducer also enables the implementation of multiplexing strategies by addressing arrays arranged in rows and columns. This fact allows to reduce the connectivity and scale up in number recording sites [7]. These features are complemented with very good mechanical conformability [11], [12], enabling flexible neural interfaces highly conformable to the brain surface. Moreover, single layer graphene prepared by chemical vapor deposition (CVD) provides optical transparency. This feature is very interesting to combine the electrical neural recordings with the recent advances in the optogenetics field [13]. Optogenetics represents a tremendous tool to remotely control and measure the electrical activity of neurons with light. It allows to selectively excite/inhibit specific cells within the densely packed neural tissue [14], providing a complementary information to electrophysiology [15]. 


\section{GRAPHENE TRANSISORS FOR NEURAL INTERFACES}

\section{A. Low frequency capabilities}

Recently, we have demonstrated the unique capabilities of active gSGFET transducers for recording infra-slow activity (ISA) [16]. This technology is able to overcome the limitations of passive electrodes in DC coupled operation, such as voltage drift, high common mode voltage, and signal attenuation due to the voltage divider between the electrode impedance and the input impedance of the amplifier [17], [18]. Along this line, there has been a particular resurgence of interest in fluctuations of ISA brain activity. The ISA signatures may serve as diagnostic, prognostic, and treatment monitoring tools for some neurological and psychological disorders [19], [20]. We have recorded epileptic seizures with some ISA features which are commonly hidden in the normal ECoG (Fig. 8). Therefore, the gSGFET technology provides a new electrophysiological tool for exploring ISA with high spatial resolution and combine the results with imaging techniques to understand its role in brain behavior (Fig. 1).

The transconductance of gSGFETs remains stable at very low frequency values, enabling the DC-coupled operation. In a gSGFET the electrode-electrolyte interface capacitance $\left(\mathrm{C}_{\mathrm{dl}}\right)$ is part of the device itself, being the input capacitance for the signal. On the other hand, when an electrode is used its impedance (mainly controlled by the $\mathrm{C}_{\mathrm{dl}}$ ) implements a voltage divider with the input impedance of the amplifier, which leads to an increasing signal attenuation at low frequencies.

\section{B. Long term in-vivo chronic evaluation}

The performance of gSGFETs in long term chronic implants has been evaluated by implanting an epicortical neural probe in the visual cortex of an adult male Wistar rat. Despite the slight decrease in the transconductance observed over several months, gSGFETs are able to record the evoked visual response with the same resolution as in the first days. Since the devices' transfer curve can be obtained in vivo, the decrease in the transconductance can be corrected in the calibration process for each recording session [16]. A shift in the Charge Neutrality Point (CNP) can be also observed over the time, which is tentatively attributed to charged molecules adsorbed on graphene surface from the biological environment. This adsorption process could also lead to a decrease in the coupling capacitance and therefore explain the observed decrease of the transconductance. Our results confirm that the implanted graphene devices are functional over 6 months of operation in vivo.

\section{ACKNOWLEDGMENT}

This work was funded by the EU Horizon 2020 programme under Grant Agreement no. 696656 (Graphene Flagship) and no. 732032 (BrainCom). This work has made use of the Spanish ICTS Network MICRONANOFABS partially supported by MINECO and the ICTS 'NANBIOSIS', more specifically by the Micro-NanoTechnology Unit of the CIBER-BBN at the IMB-CNM.

\section{REFERENCES}

[1] J. A. Assad et al., "Brain Function: Novel Technologies Driving Novel Understanding," in Bioinspired Approaches for Human-Centric Technologies, R. Cingolani, Ed. Cham: Springer International Publishing, 2014, pp. 299-334.

[2] G. Buzsáki, C. A. Anastassiou, and C. Koch, "The origin of extracellular fields and currents - EEG, ECoG, LFP and spikes," Nat. Rev. Neurosci., vol. 13, no. 6, pp. 407-420, Jun. 2012.

[3] J. Hofmeijer, C. R. van Kaam, B. van de Werff, S. E. Vermeer, M. C. Tjepkema-Cloostermans, and M. J. A. M. van Putten, "Detecting Cortical Spreading Depolarization with Full Band Scalp Electroencephalography: An Illusion?," Front. Neurol., vol. 9, 2018.

[4] D. Khodagholy et al., "NeuroGrid: recording action potentials from the surface of the brain," Nat. Neurosci., vol. 18, no. 2, pp. 310-315, Feb. 2015.

[5] J. J. Jun et al., "Fully integrated silicon probes for high-density recording of neural activity," Nature, vol. 551, no. 7679, pp. 232-236, Nov. 2017.

[6] M. E. J. Obien, K. Deligkaris, T. Bullmann, D. J. Bakkum, and U. Frey, "Revealing neuronal function through microelectrode array recordings," Syst. Biol., vol. 8, p. 423, 2015.

[7] J. Viventi et al., "Flexible, foldable, actively multiplexed, high-density electrode array for mapping brain activity in vivo," Nat. Neurosci., vol. 14, no. 12, pp. 1599-1605, Dec. 2011.

[8] L. Banszerus et al., "Ultrahigh-mobility graphene devices from chemical vapor deposition on reusable copper," Sci. Adv., vol. 1, no. 6, Jul. 2015.

[9] T. C. Cristarella, A. J. Chinderle, J. Hui, and J. Rodríguez-López, "Single-Layer Graphene as a Stable and Transparent Electrode for Nonaqueous Radical Annihilation Electrogenerated Chemiluminescence," Langmuir, vol. 31, no. 13, pp. 3999-4007, Apr. 2015.

[10] S. Drieschner et al., "Frequency response of electrolyte-gated graphene electrodes and transistors," J. Phys. Appl. Phys., vol. 50, no. 9, p. 095304, Feb. 2017.

[11] C. Hébert et al., "Flexible Graphene Solution-Gated Field-Effect Transistors: Efficient Transducers for Micro-Electrocorticography," Adv. Funct. Mater., vol. 28, no. 12, p. 1703976, 2018.

[12] B. M. Blaschke et al., "Mapping brain activity with flexible graphene micro-transistors," 2D Mater., vol. 4, no. 2, p. 025040, 2017.

[13] D. Kuzum et al., "Transparent and flexible low noise graphene electrodes for simultaneous electrophysiology and neuroimaging," Nat. Commun., vol. 5, p. 5259, Oct. 2014.

[14] W. Yang and R. Yuste, "In vivo imaging of neural activity," Nat. Methods, vol. 14, no. 4, pp. 349-359, Apr. 2017.

[15] L. F. Rossi, R. C. Wykes, D. M. Kullmann, and M. Carandini, "Focal cortical seizures start as standing waves and propagate respecting homotopic connectivity," Nat. Commun., vol. 8, no. 1, p. 217, Aug. 2017.

[16] E. Masvidal-Codina et al., "High-resolution mapping of infraslow cortical brain activity enabled by graphene microtransistors," Nat. Mater., vol. 18, no. 3, p. 280, Mar. 2019.

[17] M. J. Nelson, P. Pouget, E. A. Nilsen, C. D. Patten, and J. D. Schall, "Review of signal distortion through metal microelectrode recording circuits and filters," J. Neurosci. Methods, vol. 169, no. 1, pp. 141-157, Mar. 2008.

[18] J. P. Dreier et al., "Recording, analysis, and interpretation of spreading depolarizations in neurointensive care: Review and recommendations of the COSBID research group," J. Cereb. Blood Flow Metab., vol. 37, no. 5, pp. 1595-1625, May 2017.

[19] S. Kovac, E.-J. Speckmann, and A. Gorji, "Uncensored EEG: The role of DC potentials in neurobiology of the brain," Prog. Neurobiol., vol. 165-167, pp. 51-65, Jun. 2018.

[20] A. Mitra et al., "Spontaneous Infra-slow Brain Activity Has Unique Spatiotemporal Dynamics and Laminar Structure," Neuron, vol. 98, no. 2, pp. 297-305.e6, Apr. 2018. 


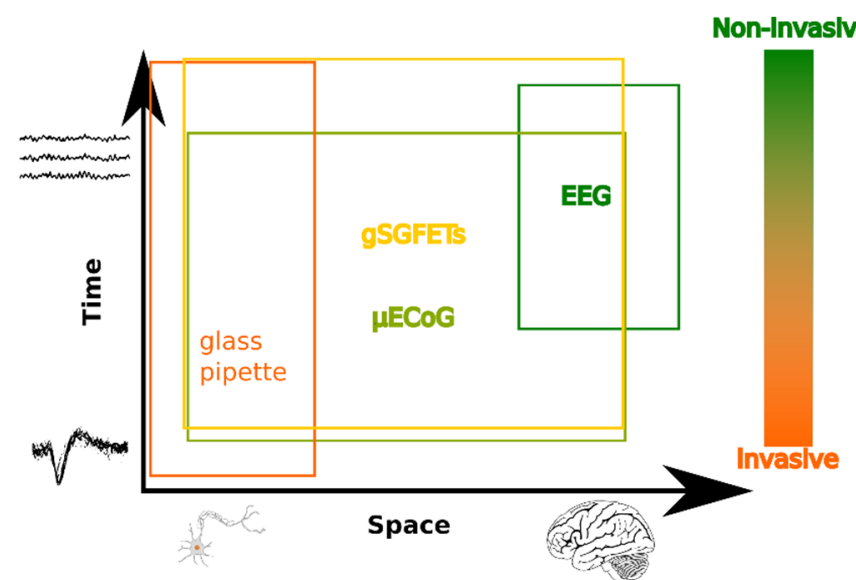

Fig. 1. Conceptual representation of the spatiotemporal regions covered by the different electrophysiological methods. gSGFETs allow $\mu E C o G$ capabilities towards infra-slow activity.
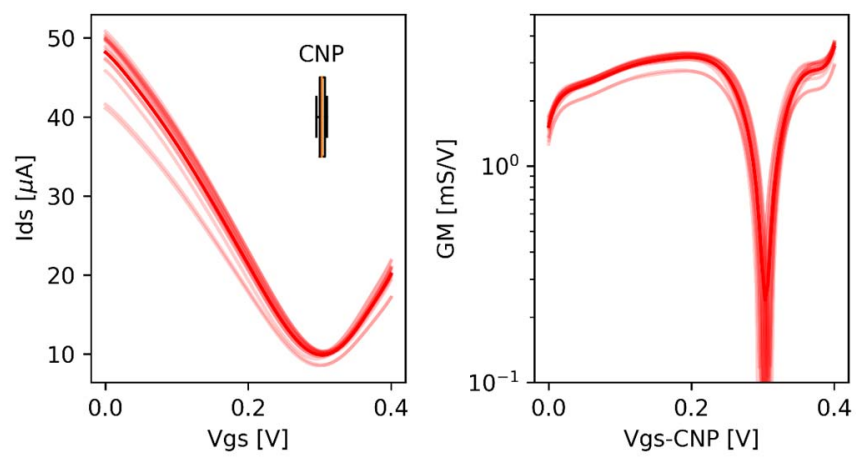

Fig. 3. Transfer curve ( $\mathrm{I}_{\mathrm{ds}} \mathrm{Vs} \mathrm{V}_{\mathrm{gs}}$ characteristics for $\mathrm{V}_{\mathrm{ds}}=50 \mathrm{mV}$ ) and normalized transconductance for 16 transistors of a $4 \times 4$ array neural probe. The boxplot inside the transfer curve represents the charge neutrality point $(\mathrm{CNP})$ for each transistor $(303 \pm 4 \mathrm{mV})$. The high homogeneity enables a single $\mathrm{V}_{\mathrm{gs}}$ bias point for all transistors.
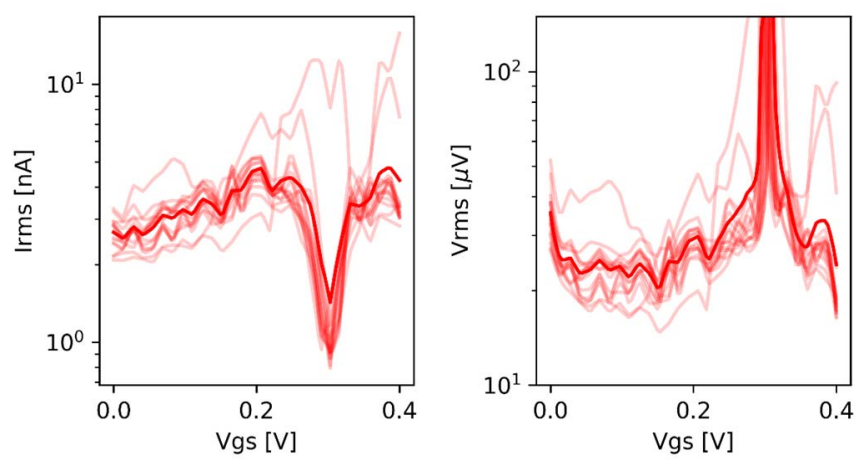

Fig. 6. RMS current noise and equivalent voltage gate noise (bandwidth $5 \mathrm{~Hz}-1 \mathrm{kHz}$ ). The equivalent voltage gate noise limits the minimum voltage which can be recorded by the transistors.
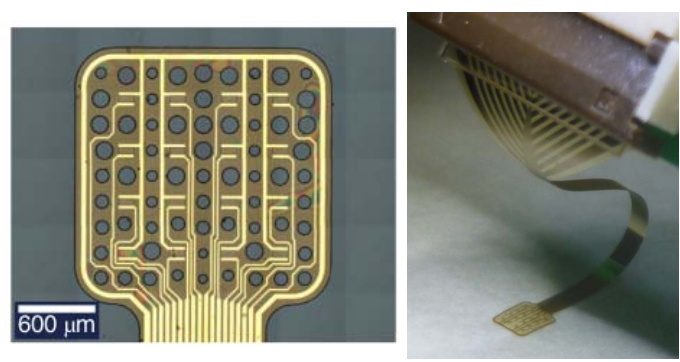

Fig. 2. Optical microscope images of the active area of a $4 \times 4$ gSGFET neural interface. Photograph of the neural probe after peeling from the wafer and introduced into a zero insertion force connector.

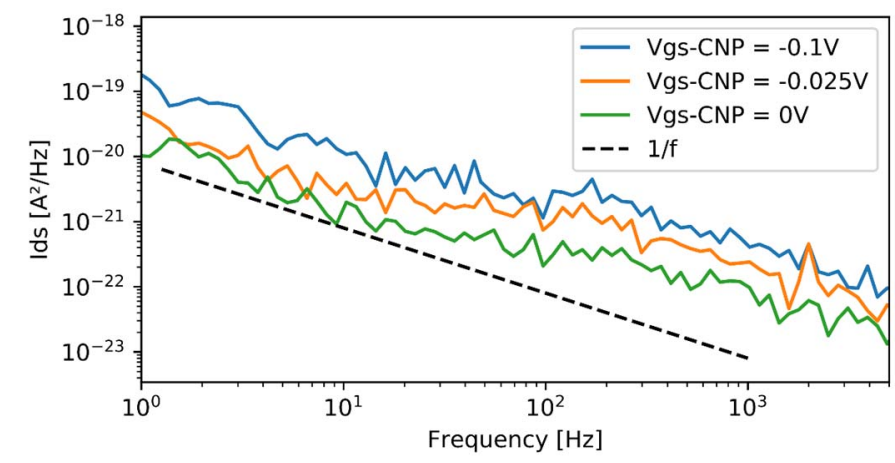

Fig. 4. Power spectral density of $\mathrm{I}_{\mathrm{ds}}$ noise at different $\mathrm{V}_{\mathrm{gs}}$ bias points. It can be observed how it depends on the $V_{\text {gs }}$ bias and shows a $1 / f$ dependence.

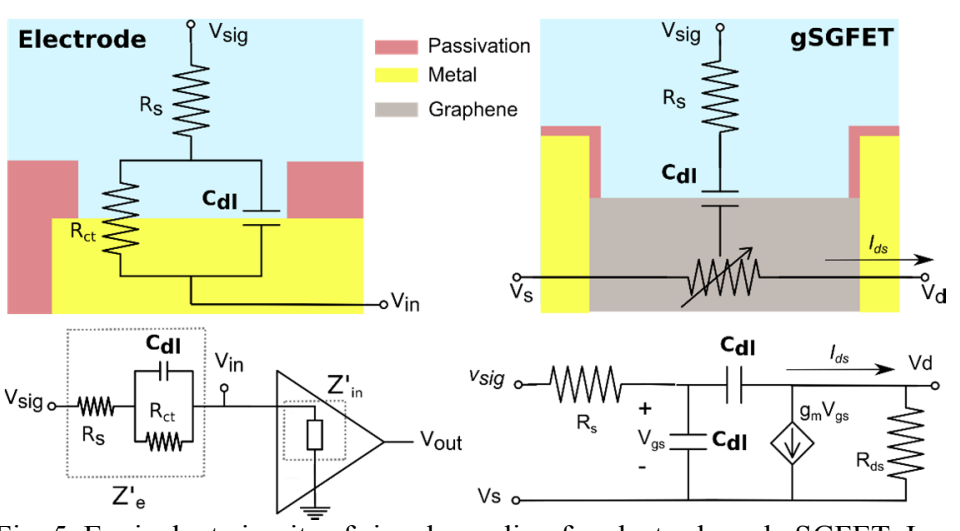

Fig. 5. Equivalent circuits of signal coupling for electrode and gSGFET. In the case of the electrode device the $\mathrm{C}_{\mathrm{dl}}$ results in a voltage divider that lead to the signal attenuation at low frequencies. This attenuation is not expected for the gSGFETs device. 

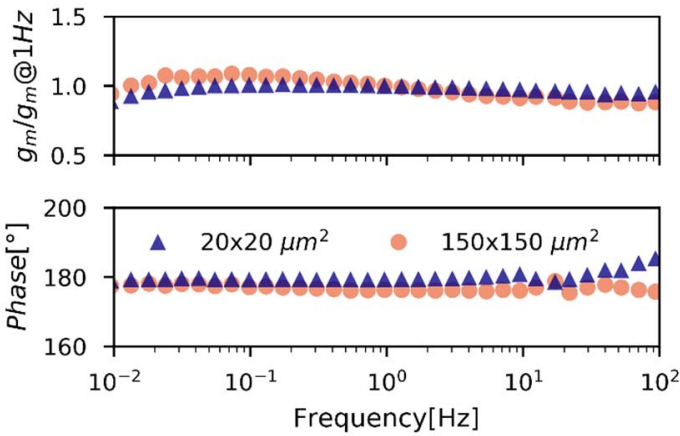

Fig. 7. Frequency response of the transconductance for two transistors sizes, showing a stable response in wide frequency range.
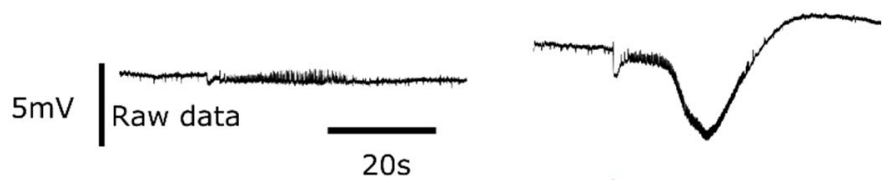

$1 \mathrm{mV}$
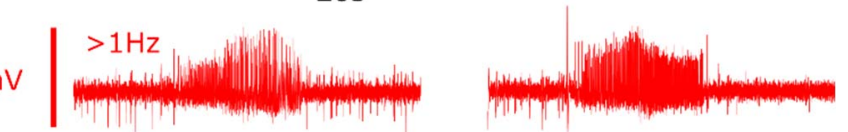

Fig. 8. Recording of two epileptic seizures induced by 4-Aminopyridine (4-AP) in wister rats. Red lines show the LFP contents of the recorded signal (both seizures present similar shape). Black lines show the fullband signal where it can be observed ISA features, which are commonly hidden in the normal ECoG.
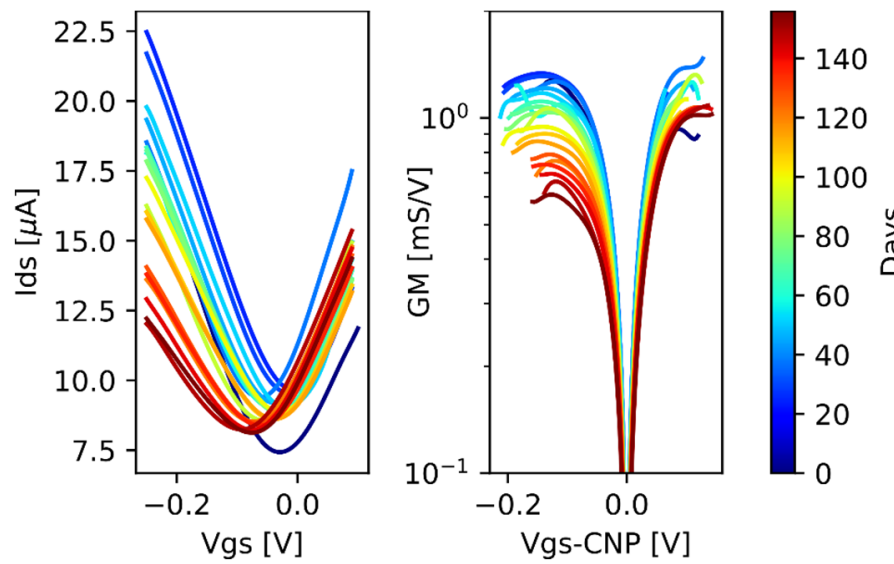

Fig. 10. Evolution of the electrical characteristics of an implanted neural interface. Lines represent the mean value of all transistors in the array and colors indicate implanted days.

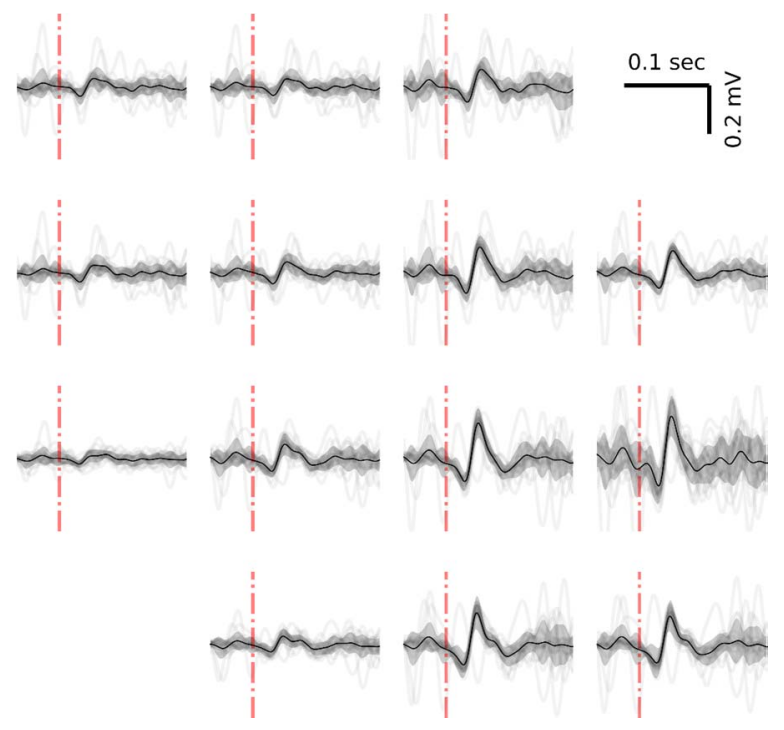

Fig. 12. Mapping of visual evoked responses used to assess the performance of the implant over time (results from a single experiment). Red vertical lines indicate the time of the LED visual stimulus, solid black lines show the average of 20 events, and gray shadow show the standard deviation.

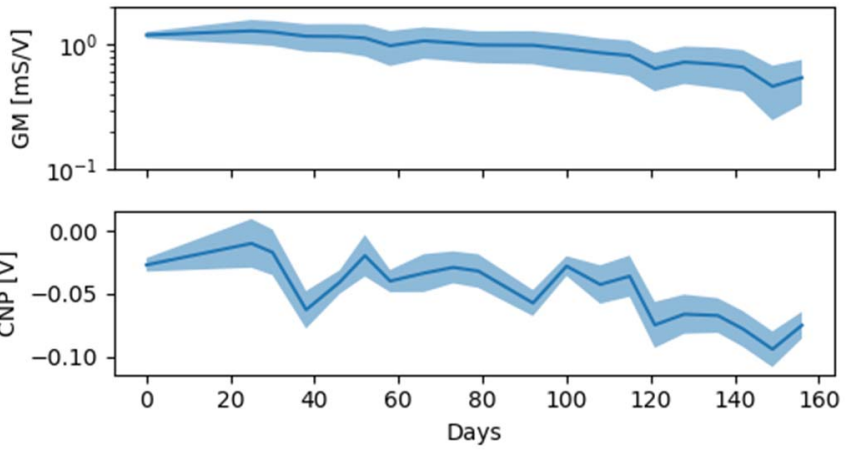

Fig. 9. Time evolution of the transconductance and CNP after implantation. Solid lines show the mean value of all transistors of the implant and shadows show the standard deviation.

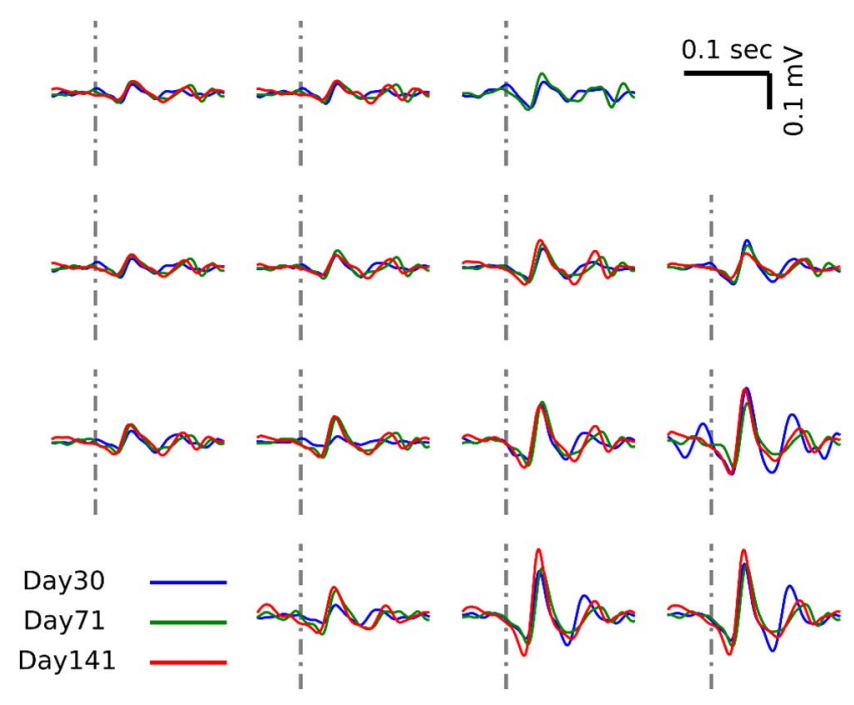

Fig. 11. Evolution of recorded visual evoked response at different days after implantation (averaged response for each experiment), demonstrating that the capability for recording evoked responses remains constant over 140 days. 